\title{
Physics and Event Selection Architecture for the ATLAS Experiment
}

\section{S. Tapprogge ${ }^{* \dagger}$}

Helsinki Institute for Physics, CERN, CH-1211 Geneva 23, Switzerland E-mail: 'Stefan. Tapprogge@cern.ch'

\begin{abstract}
The extraction of tiny signals of interest at LHC from a huge number of events is an unprecedented challenge in data handling. The ATLAS on-line system, based on three levels of selection, will provide a large and accurate data reduction in order to ease the off-line physics analysis. After the first level trigger, a reduction factor of about $10^{3}$ is to be provided by the High-Level Triggers (HLT) system, with two components: the second level trigger (LVL2) and the Event Filter (EF). LVL2 has to provide a fast decision with specially devised algorithms, acting only on a fraction of the full event, however with full granularity and combining different sub-detectors. The EF is capable of full event reconstruction: its algorithms are off-line inspired and use detailed calibration and alignment parameters. The Physics and Event Selection Architecture is presented, specifying the strategy for effectively selecting events and covering both the physics and the system performance.
\end{abstract}

\section{Introduction}

This contribution starts with a short overview of the LHC machine, its physics program and a description of the ATLAS detector. The next section contains the presentation of the ATLAS Trigger and DAQ system, which is followed by a discussion of the Physics and Event Selection Strategy. Before concluding, the selection performance is described in detail for two examples.

\subsection{The LHC machine}

The LHC will provide proton-proton collisions at $\sqrt{s}=14 \mathrm{TeV}$ for a design luminosity of $\mathcal{L}=10^{34} \mathrm{~cm}^{-2} \mathrm{~s}^{-1}$. Proton bunches will collide every $25 \mathrm{~ns}$. Due to the large inelastic $p p$ cross-section, each bunch crossing will contain at design luminosity about 23 inelastic interactions ("minimum bias events").

${ }^{*}$ Speaker.

${ }^{\dagger}$ On behalf of the ATLAS T/DAQ group. 


\subsection{The LHC physics program}

The main focus of the LHC physics is the understanding of the origin of the electroweak symmetry breaking and the search for new physics beyond the Standard Model. In addition, precision measurements of Standard Model processes (and also of new physics processes if found) will be performed.

The LHC can discover a Standard Model Higgs boson over the full relevant mass range and determine several of its properties. In case of a minimal super-symmetric extension (MSSM) of the Standard Model, at least one of the five Higgs bosons will be observed over the full parameter space. Supersymmetric particles (esp. squarks and gluinos) can be seen up to masses of about $2.5 \mathrm{TeV}$, as well as new heavy gauge bosons such as $W^{\prime}\left(Z^{\prime}\right)$ for masses less than 4.5(6) TeV. In addition, there will be several precision measurements of Standard Model parameters, such as the $W$ boson mass to an accuracy of $15 \mathrm{MeV}$ and the top quark mass to $1.5 \mathrm{GeV}$. A complete overview of the physics reach and the precision can be found in [1]

The LHC physics programme implies a huge range in the cross-sections for signal processes. This requires a very efficient on-line selection being able to also detect rare processes, whilst at the same time being able to reject background processes with larger cross-sections. The selection is going to be based mostly on high $p_{T}$ objects (where $p_{T}=$ $\mathcal{O}(10 \mathrm{GeV})$ ), such as charged leptons, photons, jets (with and without a $b$ tag) and missing transverse energy.

\subsection{The ATLAS detector}

ATLAS[2] is one of the two general-purpose experiments at LHC. It consists of a set of tracking detectors ("Inner Detector") located inside a $2 \mathrm{~T}$ solenoidal field. The interaction point is surrounded by silicon pixel layers, followed by silicon strip layers and a transition radiation tracker (TRT). Outside of the superconducting coil, a fine grained liquid Argon (LAr) electro-magnetic calorimeter is situated, followed by hadronic calorimetry: in the barrel region a scintilator-tile based and in the endcap/forward region a LAr based component. The detection and measurement of muons is performed by a stand-alone muon system based on air-core toroid magnets and precision chambers.

\section{The ATLAS Trigger and DAQ system}

In Fig.11; an overview of the ATLAS Trigger and DAQ system is given. It consists of three physical levels of on-line selection: the first level trigger (LVL1), the second level trigger (LVL2) and the event filter (EF). LVL1 has to reduce the interaction rate of $1 \mathrm{GHz}$ to about $75(100) \mathrm{kHz}$. A further factor of about 1000 in rate reduction is to be provided by the High-Level Triggers (HLT), which comprise LVL2 and the EF.

During the LVL1 decision latency, the data are stored in front-end electronic pipeline memories. Upon a LVL1 accept, these data are transferred via readout drivers to readout buffers (ROB). Here they are kept until the LVL2 decision is made. Upon an accept, the event fragments are assembled from the large number $(\approx 1700)$ of ROBs ("event building") 
and transferred to a processor of the EF farm, before being possibly accepted for mass storage.

\subsection{The LVL1 Trigger}

The decision of the LVL1 trigger [3i] is based on calorimetry (in coarse granularity) and on dedicated muon trigger chambers. It recognises signatures such e.m. clusters (to select photons and electrons), tau hadrons, jets, missing (as well as summed) transverse energy and muons.

LVL1 operates within a maximum latency of $2.5 \mu \mathrm{s}$ and is based on special purpose hardware, using custom designed chips (ASICs) and programmable logic arrays (FPGAs). It also provides guidance to the next stage (LVL2) about regions in the detector where high $p_{T}$ objects are

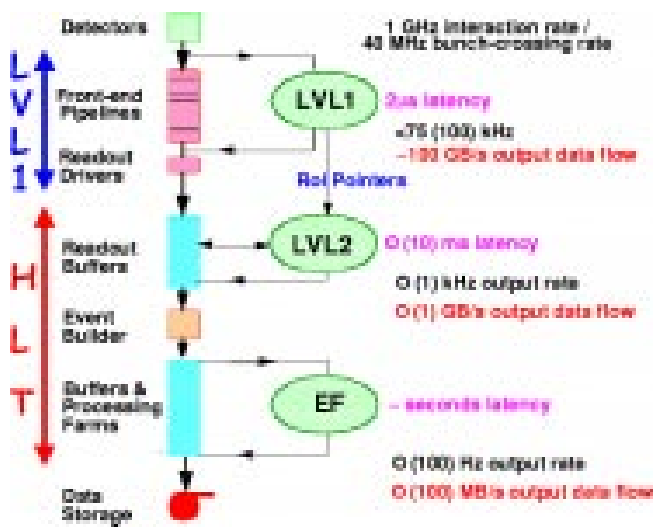

Figure 1: Overview of the ATLAS Trigger and DAQ system. located (so called "Regions of Interest" (RoI)).

\subsection{The HLT system}

The LVL2 component of the HLT [4] will use mostly only a few percent of the full event data, thanks to the guidance obtained through the RoI information provided by LVL1. It has access to the full granularity information from all sub-detectors and thus can combine e.g. calorimeter and tracking information. LVL2 has to make its decision within a latency of about $10 \mathrm{~ms}$.

After event building, the full event information is available for the EF processing, which will also have access to more detailed calibration and alignment information. The latency requirements are less strict than for LVL2: the average time for an event decision should be about $1 \mathrm{~s}$.

Both components of the HLT will perform a selection based on algorithms implemented in software, thus providing a very flexible approach to cope with changes in conditions. For LVL2, the development of optimised code is foreseen, whereas at the EF off-line based code should be deployed.

\section{Physics and Event Selection Strategy}

The physics and event selection strategy has to be flexible, redundant and robust in order to cope with the unprecedented conditions at LHC. Four major elements define the selection strategy. These are:

Algorithms, which identify physics objects with associated properties and global features of the event (e.g. an electron candidate with its values of $E_{T}, \eta$ and $\phi$, as well as information on its isolation), 
Sequence, which defines the order of the execution of algorithms (e.g. according to the complexity of the algorithms),

Selection, which performs the rejection of background events (by executing sequences of algorithms and verifying the physics objects obtained against a trigger menu),

Classification, which identify basic event topologies and tag events for later analysis (e.g. for a hot-line path to quickly analyse events of special interest), but will not reject any event.

The strategy has been derived based on the physics program of ATLAS, defining the physics objects and the criteria for selection, which are mostly using inclusive signatures to maximise the discovery potential. Many algorithms have then been implemented to perform this selection and the selection sequence has been optimised for the best system performance.

\subsection{Selection criteria}

A rather small set of inclusive physics signatures (i.e. requiring one or two high $p_{T}$ physics objects) will cover most of the physics program of ATLAS. The selection uses (in case of low luminosity $\left(\mathcal{L}=10^{33} \mathrm{~cm}^{-2} \mathrm{~s}^{-1}\right)$ the following signatures ${ }^{1}: e 25 i, 2 e 15 i, \gamma 60 i, 2 \gamma 20 i$, $\mu 20 i, j 360,3 j 150,4 j 100$ and $j 60+x E 60$. For low luminosity an additional selection is made to be able to do $B$-hadron physics (see below).

\subsection{Selection software design}

The software to perform the selection and control of the HLT system is being developed in a $\mathrm{C}++/ \mathrm{OO}$ approach, using a mature software development process. The requirements capture has been followed by a high-level design. Fig. $\overline{2}$ shows the domain decomposition obtained, highlighting the three major areas: the Steering controls the execution of the selection processes, the HLT algorithms extract the physics objects candidates with their properties from raw data and the Event Data Model defines the entities used in the processing and their relationship. These domains have several external interfaces to provide access to raw and meta data, to monitoring, to

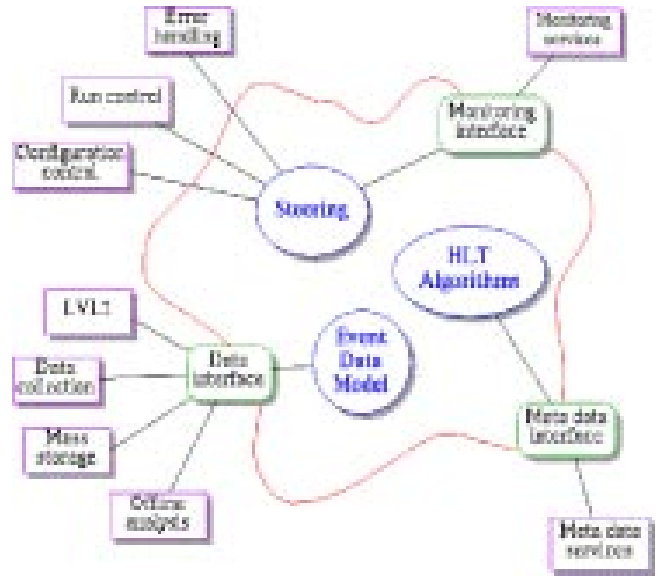

Figure 2: Domain decomposition of the selection and control software. configuration and to other services. The proper definition of these interfaces will allow the major part of the software to be used simultanously on-line as well as off-line (e.g. for testing and development).

The on-going steps involve the detailed design, the implementation and the testing of the package.

\footnotetext{
${ }^{1}$ Here the first letter indicates the type of the object, the number gives the $p_{T}$ threshold and $i$ indicates an isolation criterium
} 


\section{Selection Performance}

Two aspects of the performance can be distinguished: the physics performance (e.g. efficiency for signal processes and rejection against background processes, which should both be as large as possible) and the system performance, quantified e.g. by the execution time needed and the amount of data required to make the selection (both should be small, but at least within certain limits).

All these parameters influence the size and the cost of the HLT system, e.g. the product of rate and data size determines the bandwidth needed and the product of rate and execution time influences the number of $\mathrm{CPU}$ required. It is important to realize that physics and system performance cannot be optimised separately. They also influence the resources needed for the off-line computing, e.g. the size of the mass storage and of the processing farms for reconstruction and analysis.

\subsection{Physics performance example 1: high $p_{T}$ electron selection}

The high $p_{T}$ electron trigger selects a variety of physics processes, such as the production of $W$ and $Z$ bosons and Drell-Yan pairs, of top quarks and of Higgs bosons, as well as e.g. new heavy gauge bosons. The challenge here is the rejection of a large background due to jets faking an electron signature.

The selection starts with a detailed shower shape analysis using the information from the fine-grained ATLAS e.m. calorimetry. It is followed by a search for charged tracks, which are then matched to the calorimeter cluster. Further refinements include the use of transition radiation signals to identify electrons and the recovery of events which have undergone Bremsstrahlung.

Fig. $\overline{3}_{1}$ shows the rate (at design luminosity) for the electron trigger vs. the efficiency (wrt. LVL1) for various

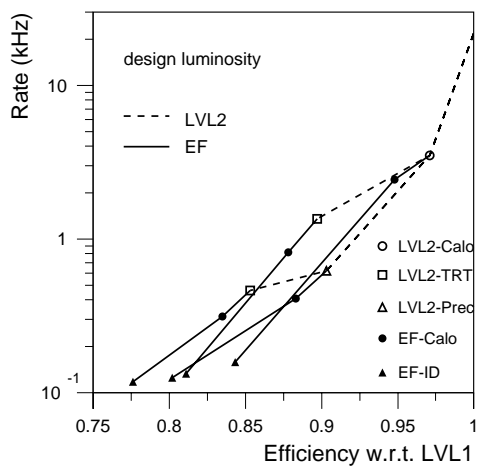

Figure 3: Rate vs. efficiency for the high $p_{T}$ electron HLT selection at design luminosity for different selection sequences. steps of various selection sequences (which differ in the sharing of the selection between LVL2 and EF). The initial LVL1 rate of $21.7 \mathrm{kHz}$ is reduced by the HLT to $114 \mathrm{~Hz}$, where the accepted sample consists of $40 \%$ of electrons from $W$ decays, $13 \%$ of electrons from $b$ and $c$ quark decays and only $47 \%$ from fake signatures or conversions.

\subsection{Physics performance example 2: $B$-physics selection}

The selection of events for $B$-physics is used to make measurements of CP violation, $B_{s}$ oscillations and rare $B$-decays. The major challenge is the need for a semi-exclusive decay reconstruction which involves low $p_{T}(\mathcal{O}(\mathrm{GeV}))$ particle, where LVL1 can not provide guidance. Thus an unguided search over the full tracking volume is required.

The $B$-physics selection is initiated at LVL1 using a low $p_{T}$ muon signature (nominal threshold of $6 \mathrm{GeV})$, which yields at low luminosity $\left(\mathcal{L}=10^{33} \mathrm{~cm}^{-2} \mathrm{~s}^{-1}\right)$ a rate of $23 \mathrm{kHz}$. The muon selection is refined at LVL2 by using the information from the precision muon 


\begin{tabular}{|c|c|c|c|c|c||r|}
\hline $\mathrm{e} 25 \mathrm{i}$ & $\gamma 60 \mathrm{i}$ & $\mu 20 \mathrm{i}$ & $\mathrm{j} 360$ & $\mathrm{j} 60+\mathrm{xE} 60$ & B-physics & total \\
$2 \mathrm{e} 15 \mathrm{i}$ & $2 \gamma 20 \mathrm{i}$ & & $3 \mathrm{j} 150$ & & & \\
& & & $4 \mathrm{j} 100$ & & & \\
\hline$\sim 20 \mathrm{~Hz}$ & $\sim 20 \mathrm{~Hz}$ & $\sim 15 \mathrm{~Hz}$ & $\sim 25 \mathrm{~Hz}$ & $\sim 20 \mathrm{~Hz}$ & $\sim 60 \mathrm{~Hz}$ & $\sim 160 \mathrm{~Hz}$ \\
\hline
\end{tabular}

Table 1: Rates after HLT selection at low luminosity.

detectors and by matching to a track found in the Inner Detector. The resulting rate is $5 \mathrm{kHz}$. Next, an unguided search for low $p_{T}$ tracks has to be performed in the Inner Detector to be able to do semi-exclusive decay reconstruction. Three decay modes are used to benchmark the performance: $B \rightarrow J / \Psi+X$ with $J / \Psi \rightarrow e^{+} e^{-}\left(\mu^{+} \mu^{-}\right), B_{d} \rightarrow \pi^{+} \pi^{-}$ and $B_{s} \rightarrow D_{s}\left(\phi\left(K^{+} K^{-}\right) \pi\right)+X$. The rate obtained after this selection amounts to $60 \mathrm{~Hz}$.

\subsection{System performance}

As mentioned above, especially LVL2 is subject to strong performance requirements (e.g. average latency of about $10 \mathrm{~ms}$ and access mostly to a few percent of the event data) in order to reduce the necessary resources needed to implement the system. A sequential selection will be used, where an event is rejected as early and as fast as possible, using algorithms with increasing complexity (and thus execution time).

Fig.'Á-1's shows a measurement of the LVL2 decision for a high $p_{T}$ electron selection, including both the latency due to the algorithms and due to the data access, using an emulated system of about 100 nodes $^{2}$. Half of the events have the decision taken within $3.1 \mathrm{~ms}$, and $99 \%$ of the events are treated within $10.8 \mathrm{~ms}$, which shows that for this selection the latency requirement is already fulfilled using today's technology.

\subsection{Total rate}

In table $i_{-1}^{1}$ an overview of the rates expected at

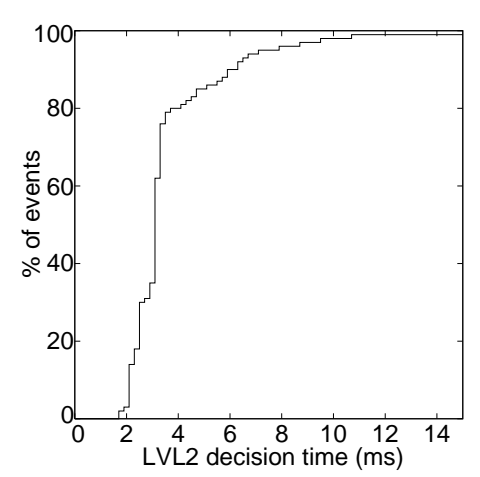

Figure 4: Integrated latency distribution for the electron selection at LVL2. The first selection step (calorimetry only) takes up to $3 \mathrm{~ms}$, the first tracking step (TRT) up to about $5 \mathrm{~ms}$ and the second tracking step (SCT/pixel) up to about $9 \mathrm{~ms}$.

$\mathcal{L}=10^{33} \mathrm{~cm}^{-2} \mathrm{~s}^{-1}$ after the HLT selection is given. Not indicated are error estimates on these rates, which are due to stastistical accuracy on one side (of up to $20 \%$ ) and due to uncertainties in the cross-sections for signal and background processes, which can be larger than the statistical accuracy in some cases.

Also it has to be noted that this compilation does not contain any pre-scaled triggers, to be used e.g. for extending cross-section measurements to smaller scales, to obtain monitor and calibration samples of physics events and to provide sets of data to understand backgrounds.

\section{Conclusions}

The on-line selection architecture for the ATLAS experiment at LHC has been presented,

\footnotetext{
${ }^{2} 450 \mathrm{MHz}$ dual Pentium II PC
} 
which is capable of reducing a $1 \mathrm{GHz}$ interaction rate to a value of $\mathcal{O}(100) \mathrm{Hz}$ for mass storage. The selection architecture provides good efficiency for the broad spectrum of LHC physics signatures.

The on-line selection has strong relations, dependencies and influences on the offline computing. On one side, the last stage (EF) of the on-line selection is to use off-line based algorithms, while on the other side the resources needed for off-line computing (CPU power, storage space) strongly depend on the quality of the on-line selection. An un-biased rejection of most events which are never going to be analysed off-line is mandatory in the LHC environment and should be achieved by mostly using simple, inclusive signatures. Tagging of events on-line (event classification) will ease the off-line analyses.

\section{References}

[1] ATLAS collaboration, ATLAS Detector and Physics Performance Technical Design Report, CERN/LHCC 99-14 and 99-15 (1999).

[2] ATLAS collaboration, ATLAS Technical Proposal, CERN/LHCC 94-43 (1994).

[3] ATLAS collaboration, ATLAS First Level Trigger Technical Design Report, CERN/LHCC 1998-14 (1998).

[4] ATLAS collaboration, ATLAS High-Level Triggers, DAQ and DCS Technical Proposal, CERN/LHCC 2000-17 (2000). 